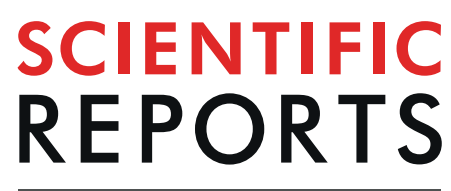

\title{
Spleen Stiffness as Predictor of Esophageal Varices in Cirrhosis of Different Etiologies
}

\author{
Carmen Fierbinteanu-Braticevici ${ }^{1,2}$, Laura Tribus ${ }^{1,2}{ }^{2}$, Razvan Peagu ${ }^{1,2}$, Ana Petrisor ${ }^{1,2}$, \\ Cristian Baicus ${ }^{3}$, Dragos Cretoiu $\mathbb{D}^{4,5^{*}}$, Artur Pasternak $\mathbb{D}^{6}$, Gabriela Oprea ${ }^{1,2}$, \\ Adina Purcareanu ${ }^{1,7}$ \& Alexandru C. Moldoveanu ${ }^{1,2}$
}

The purpose of this study is to determine whether esophageal varices (EV) can be identified through the evaluation of spleen stiffness (SSM) via acoustic radiation force impulse (ARFI). A total of 135 patients suffering from cirrhosis underwent a clinical exam, laboratory tests, abdominal ultrasound, liver stiffness (LSM) measurement, SSM evaluation and upper gastrointestinal endoscopy. Based on the endoscopy results, the patients were classified into three groups: those with no evident $\mathrm{EV}$, those with small EV and those with varices needing treatment (VNT). Patients with EV of any grade had significantly higher average SSM values over those with no EV $(3.37 \mathrm{~m} / \mathrm{s}$ versus $2.79 \mathrm{~m} / \mathrm{s}$, $\mathrm{p}$-value $<0.001)$, while patients with VNT showed an even greater difference $(3.96 \mathrm{~m} / \mathrm{s}$ versus $2.93 \mathrm{~m} / \mathrm{s}$, $\mathrm{p}$-value $<0.001)$. SSM proved to be an excellent method of predicting patients with VNT.

Currently, patients suffering from cirrhosis are recommended upper gastrointestinal endoscopy (UGE) screening, in order to detect esophageal varices (EV). Unfortunately, UGE is an expensive, time-consuming method that is not well tolerated by patients. Consequently, various non-invasive methods of diagnosing EV have recently been proposed, the most promising one being the measuring of spleen stiffness (SSM). Our study proved that SSM, measured through acoustic radiation force impulse elastography, can consistently predict large EVs in cirrhotic patients, including those with a high risk of bleeding. Those with a higher bleeding risk are currently categorized as varices needing treatment (VNT) in the expanded Baveno VI guidelines. Our study proposes SSM as an alternative method for ruling out patients with VNT. This avoids the need for upper GI endoscopy, thus improving upon the Baveno VI criteria.

Portal hypertension $(\mathrm{PH})$ as well as esophageal varices are major aggravations of hepatic cirrhosis, which are associated with increased mortality rates ${ }^{1,2}$. The gold standard method for measuring PH is the hepatic pressure venous gradient (HPVG), whilst the diagnosis of EV requires upper endoscopy ${ }^{3,4}$. Both of these investigations are invasive, not always well tolerated by patients, expensive, difficult to repeat and HPVG is also not widely available. Therefore, the need for noninvasive tools that accurately predict the presence and severity of $\mathrm{PH}$ and EV is crucial to help perform a proper prophylaxis of variceal bleeding ${ }^{5-7}$.

$\mathrm{PH}$ leads to splenic congestion, which induces architectural changes in the splenic arteries and veins, resulting in fibrosis and an increase in spleen stiffness (SSM $)^{5,8}$. Recently, noninvasive techniques that measure SSM, in order to identify EVs and their hemorrhage risk are gaining more and more interest ${ }^{5,7,9-12}$. SSM can be evaluated through elastography techniques, such as shear wave elastography (SWE) $)^{13}$, transient elastography $(\mathrm{TE})^{14}$, strain elastography and acoustic radiation force impulse (ARFI $)^{15}$. These techniques are accurate in predicting the existence of EVs in most cases of cirrhosis with portal hypertension, including both viral and alcoholic cirrhosis ${ }^{16-19}$. TE is a useful method to detect high risk EVs, although it is limited by factors such as obesity and the ascites ${ }^{5,20}$. SWE is another good diagnostic method for those with clinically significant $\mathrm{PH}^{21,22}$.

\footnotetext{
${ }^{1}$ Medical Clinic II and Gastroenterology, Carol Davila University of Medicine and Pharmacy, Bucharest, 050474, Romania. ${ }^{2}$ Department of Gastroenterology, Bucharest Emergency University Hospital, Bucharest, 050098, Romania. ${ }^{3}$ Department of Clinical Epidemiology and Biostatistics, Carol Davila University of Medicine and Pharmacy Bucharest, Bucharest, 050474, Romania. ${ }^{4}$ Department of Cellular and Molecular Biology and Histology, Carol Davila University of Medicine and Pharmacy, Bucharest, 050474, Romania. ${ }^{5}$ Fetal Medicine Excellence Research Center, Alessandrescu-Rusescu National Institute for Mother and Child Health, Bucharest, 011062, Romania. ${ }^{6}$ Department of Anatomy, Jagiellonian University Medical College, Krakow, 31-008, Poland. ${ }^{7}$ Department of Internal Medicine, Bucharest Emergency University Hospital, Bucharest, 050098, Romania. *email: dragos@cretoiu.ro
} 
Nevertheless, one of the most studied elastography techniques is ARFI, a new alternative investigation that evaluates tissue elasticity and that is reliable even in cases associating obesity or ascites ${ }^{5,6}$. ARFI is categorized as a displacement imaging technique, which uses a deep focused radiation force, induced by ultrasound. This measures the stiffness of a tissue, both quantitatively and qualitatively. The displacement is measured by comparing the locations of tissue echoes emitted before and immediately after the impulse ${ }^{15}$. Our study aimed to assess the clinical use of splenic stiffness (SSM), evaluated through ARFI, in patients suffering from cirrhosis. This was conducted in order to predict the existence and gravity of esophageal varices.

\section{Materials and Methods}

Study design. Our prospective study was performed on a series of patients with compensated cirrhosis from the Gastroenterology Department at the Bucharest Emergency University Hospital, between October 2016 and September 2017. The exclusion criteria included: decompensated cirrhosis (as evaluated by the presence of ascites, hepatic encephalopathy and the Child-Pugh Score), current treatment with beta blockers and previous or current variceal bleeding. The diagnosis of cirrhosis was established based on a combination of results from the clinical exam, laboratory blood tests, abdominal ultrasound and upper gastrointestinal endoscopy. Either positive histology from hepatic biopsy or LSM $>10 \mathrm{kPa}$ on TE were required as inclusion criteria.

A study protocol was respected for each patient, which included a clinical exam, laboratory tests (hemogram, hepatic panel, serum albumin), abdominal ultrasound (highlighting the spleen diameter, portal vein diameter as well as the existence of ascites), liver and spleen ARFI elastography and upper gastrointestinal endoscopy. All tests were conducted within a maximum of 72 hours from each other. All methods were performed in accordance with the relevant institutional and national guidelines and regulations ${ }^{23,24}$. The study was approved by the Local Ethics Committee and informed consent forms were signed by each patient.

Elastography measurement. Acoustic Radiation Force Imaging (ARFI) elastography was performed on each patient, after at least 12 hours of fasting, using the Siemens Acuson S2000 (Siemens AG) device and a dual $\mathrm{B}$-mode ultrasound and quantitative elastography convex probe (Siemens $4 \mathrm{C} 1$ ). In order to conduct the measurements each patient was asked to hold his or her breath for 5 seconds. We carried out two separate sets of measurements - LSM and SSM respectively. Measurement sites were chosen $2 \mathrm{~cm}$ beneath the capsule of each organ. Each set of measurements was repeated at least ten times and the average value was chosen.

Esophageal varices classification. Esophageal varices were evaluated for each patient, using upper gastrointestinal endoscopy and were classified into three groups, according to the expanded Baveno VI criteria ${ }^{25}$. Group 1 included patients with no esophageal varices, Group 2 - patients with low risk esophageal varices (varices that had a thickness of less than $5 \mathrm{~mm}$ ) and Group 3 - patients with varices needing treatment (VNT - either large esophageal varices that had a thickness of more than $5 \mathrm{~mm}$ or varices displaying any signs of a high risk of bleeding: red wales, cherry red spots).

Statistical analysis. The results were inputted into a table using Microsoft Excel (Microsoft Corporation) and analyzed using Microsoft Excel, SPSS Version 23 (IBM Corporation) and MedCalc 19. The normality of the variables was assessed using histograms and the Shapiro-Wilk test. Univariate analysis for parameters with normal distribution was assessed using Independent Samples T Test, while for the parameters without normal distribution the Independent Samples Mann-Whitney U Test (for two categories) and Independent Samples Kruskal-Wallis Test (for more than two categories) were used. Multivariate analysis was performed using binary logistic regression. For each parameter, we assessed not only sensitivity and specificity, but also positive and negative predictive values, and positive and negative likelihood ratios at three distinct cut-off points - a cut-off based on the highest Youden's J statistic point, a rule out cut-off (best negative likelihood ratio) and a rule in cut-off (best positive likelihood ratio). Cut-off values were selected based on the ROC curve and overall accuracy was determined using the area under the curve (AUROC). The strength of associations was calculated using the Spearman Rho test and multivariate analysis was performed on all parameters associated in univariate analysis. A p-value of $<0.05$ was considered statistically significant for all tests. The area under the ROC curve was interpreted as insignificant for results between $0.500-0.600$, a poor predictor for results $0.600-0.700$, a fair predictor for results $0.700-0.800$, a good predictor for results $0.800-0.900$ and an excellent predictor for results $>0.900$. The comparison between ROC curves was performed using the DeLong test, as illustrated in Fig. 1.

\section{Results}

Characteristics of patients. The study included a total number of 135 patients, out of which 77 were male and 58 were female. The patients were evenly distributed by age and gender, with no statistical difference between the groups. The etiology of cirrhosis was alcoholic (33 patients), hepatitis B (20 patients), hepatitis C (76), cryptogenic (5 patients) and primary biliary cirrhosis (1 patient). All enrolled patients were included in the study. Bivariate analysis identified several factors that were associated with the size of varices, including aspartate aminotransferase $(\mathrm{p}=0.001)$, platelet count $(\mathrm{p}<0.001)$, spleen diameter $(\mathrm{p}<0.001)$, portal vein diameter $(\mathrm{p}<0.001)$, liver elastography $(\mathrm{p}<0.001)$ and spleen elastography $(\mathrm{p}<0.001)$, as listed in Table 1 .

Diagnostic performance of spleen stiffness. There is a stepwise increase in spleen stiffness values correlated with an increased severity of portal hypertension. Patients with esophageal varices of any grade had significantly higher average spleen elastography values than those with no varices $(3.37 \mathrm{~m} / \mathrm{s}$ versus $2.79 \mathrm{~m} / \mathrm{s}$, $\mathrm{p}$-value $<0.001)$, while those with varices needing treatment had an even greater difference versus patients with no or small varices $(3.96 \mathrm{~m} / \mathrm{s}$ versus $2.93 \mathrm{~m} / \mathrm{s}$, p-value $<0.001)$. AUROC values were fair for the detection of any degree of esophageal varices (AUROC $=0.776,95 \% \mathrm{CI}=0.698-0.853, \mathrm{p}<0.001$ ) and excellent at distinguishing patients with varices needing treatment $(A U R O C=0.972,95 \% \mathrm{CI}=0.944-1.000, \mathrm{p}<0.001)$. The results of 


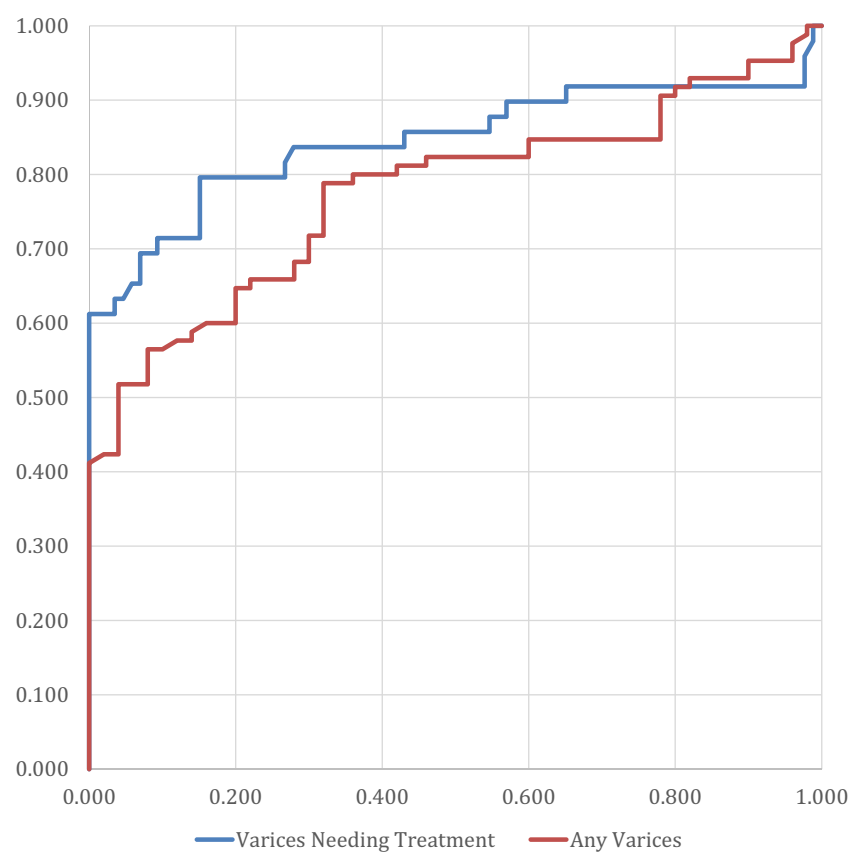

Figure 1. ROC curves for spleen elastography.

\begin{tabular}{|l|l|l|l|l|}
\hline & $\begin{array}{l}\text { No Varices } \\
(\mathbf{n = 5 0 , 4 4})\end{array}$ & $\begin{array}{l}\text { Small Varices } \\
(\mathbf{n = 3 6 , 3 1 \% )}\end{array}$ & $\begin{array}{l}\text { Varices Needing } \\
\text { Treatment } \mathbf{n = 2 9 , 2 5 \% )}\end{array}$ & p-value \\
\hline Age & $60.16 \pm 10.16$ & $57.61 \pm 7.14$ & $62.89 \pm 9.82$ & 0.091 \\
\hline Gender & $23 \mathrm{~F}$ & $\begin{array}{l}14 \mathrm{~F} \\
22 \mathrm{M}\end{array}$ & $\begin{array}{l}14 \mathrm{~F} \\
15 \mathrm{M}\end{array}$ & 0.733 \\
\hline Aspartate Aminotransferase & $51.36 \pm 38.21$ & $48.44 \pm 24.30$ & $69.79 \pm 26.95$ & 0.001 \\
\hline Alanine Aminotransferase & $44.66 \pm 21.86$ & $53.5 \pm 28.81$ & $56.51 \pm 32.25$ & 0.347 \\
\hline Platelet Count & $188082 \pm 70432$ & $155958 \pm 88680$ & $102175 \pm 47657$ & $<0.001$ \\
\hline Spleen Diameter & $110.96 \pm 19.67$ & $126.69 \pm 22.58$ & $141.69 \pm 22.74$ & $<0.001$ \\
\hline Portal Vein Diameter & $10.16 \pm 1.96$ & $10.49 \pm 1.68$ & $11.69 \pm 1.84$ & $<0.001$ \\
\hline Hemoglobin & $13.16 \pm 1.25$ & $13.28 \pm 1.17$ & $12.33 \pm 1.79$ & 0.210 \\
\hline Liver Elastography & $2.00 \pm 0.51$ & $2.19 \pm 0.54$ & $2.55 \pm 0.57$ & $<0.001$ \\
\hline Spleen Elastography & $\mathbf{2 . 7 9} \pm \mathbf{0 . 4 1}$ & $\mathbf{3 . 0 2} \pm \mathbf{0 . 4 0}$ & $\mathbf{3 . 9 6} \pm \mathbf{0 . 3 7}$ & $<\mathbf{0 . 0 0 1}$ \\
\hline
\end{tabular}

Table 1. Patient characteristics and univariate analysis.

the liver elastography were poor for the detection of any degree of esophageal varices (AUROC $=0.682,95 \%$ $\mathrm{CI}=0.586-0.777, \mathrm{p}<0.001)$ and fair for distinguishing patients with varices needing treatment $(\mathrm{AUROC}=0$. $712,95 \% \mathrm{CI}=0.613-0.812, \mathrm{p}<0.001)$. The results of the splenic elastography were superior for varices needing treatment $(p<0.0001)$, but were not statistically significant for the diagnosis of any varices $(p=0.0768)$. The accuracy of spleen elastography for each test is detailed further in Table 2. The Spearman correlation test showed a stronger association for spleen elastography $(r=0.461, p<0.001$ for any grades of varices and $r=0.671, p<0.001$ for varices needing treatment) than any other investigated parameters, including liver elastography (which was second best, with a more modest $r=0.304, p<0.001$ for any grades of varices and $r=0.302, p<0.001$ for varices needing treatment). In the case of varices needing treatment, the variation of accuracy depending on prevalence is illustrated in Table 3. The multivariate analysis showed that spleen elastography was the sole parameter that was precisely correlated not only with the signs of an elevated risk of bleeding, but also with the existence of varices, irrespective of grade ( $p$-values are presented in Table 4). The various etiologies of cirrhosis did not influence the spleen elastography results in our study (Table 5).

\section{Discussions}

One of the major complications of cirrhosis, regardless of its etiology is portal hypertension, a frequent cause of death for cirrhotic patients. Clinically significant portal hypertension (CSPH, hepatic venous pressure gradient $\geq 10 \mathrm{mmHg}$ ) is the main cause of cirrhosis decompensation and it can influence the survival of cirrhotic patients. The complications of CSPH include ascites, hepatic encephalopathy and bleeding from gastroesophageal varices, which is still associated with a high mortality rate of $10-20 \%{ }^{4}$ at six weeks. Therefore, the assessment 


\begin{tabular}{|c|c|c|}
\hline & $\begin{array}{l}\text { Any Esophageal } \\
\text { Varices }\end{array}$ & $\begin{array}{l}\text { Varices Needing } \\
\text { Treatment (VNT) }\end{array}$ \\
\hline AUROC (95\% CI) & $0.776(0.698-0.853)$ & $0.972(0.944-1.000)$ \\
\hline Balanced Cut-off & $\begin{array}{l}3 \mathrm{~m} / \mathrm{s}: \\
79 \% \text { Sensitivity; } \\
67 \% \text { Specificity; } \\
80 \% \text { PPV; } \\
65 \% \text { NPV; } \\
2.39 \text { LR +; } \\
0.31 \text { LR-. }\end{array}$ & $\begin{array}{l}3.5 \mathrm{~m} / \mathrm{s}: \\
\text { 93\% Sensitivity; } \\
\text { 86\% Specificity; } \\
64 \% \text { PPV; } \\
\text { 98\% NPV; } \\
\text { 6.64 LR+; } \\
0.08 \text { LR-. }\end{array}$ \\
\hline Rule In Cut-off & $\begin{array}{l}3.5 \mathrm{~m} / \mathrm{s}: \\
\text { 47\% Sensitivity; } \\
\text { 96\% Specificity; } \\
80 \% \text { PPV; } \\
65 \% \text { NPV; } \\
\text { 11.75 LR+; } \\
\text { 0.55 LR-. }\end{array}$ & $\begin{array}{l}3.8 \mathrm{~m} / \mathrm{s}: \\
55 \% \text { Sensitivity; } \\
98 \% \text { Specificity; } \\
\text { 89\% PPV; } \\
\text { 89\% NPV; } \\
\text { 27.5 LR+; } \\
\text { 0.46 LR-. }\end{array}$ \\
\hline Rule Out Cut-off & $\begin{array}{l}2.5 \mathrm{~m} / \mathrm{s}: \\
92 \% \text { Sensitivity; } \\
22 \% \text { Specificity; } \\
66 \% \text { PPV; } \\
61 \% \text { NPV; } \\
1.18 \text { LR+; } \\
0.36 \text { LR-. }\end{array}$ & $\begin{array}{l}3.2 \mathrm{~m} / \mathrm{s}: \\
\text { 97\% Sensitivity; } \\
\text { 69\% Specificity; } \\
\text { 46\% PPV; } \\
\text { 99\% NPV; } \\
3.13 \mathrm{LR}+ \\
0.04 \text { LR-. }\end{array}$ \\
\hline Average Values & $3.37 \mathrm{~m} / \mathrm{s}$ & $3.96 \mathrm{~m} / \mathrm{s}$ \\
\hline
\end{tabular}

Table 2. The accuracy of spleen elastography.

\begin{tabular}{|l|l|l|l|l|}
\hline Prevalence & Sensitivity & Specificity & PPV & NPV \\
\hline $50.0 \%$ & $93.1 \%$ & $0.0 \%$ & $73.0 \%$ & $0.0 \%$ \\
\hline $40.0 \%$ & $93.1 \%$ & $0.0 \%$ & $64.3 \%$ & $0.0 \%$ \\
\hline $30.0 \%$ & $93.1 \%$ & $31.8 \%$ & $64.3 \%$ & $77.8 \%$ \\
\hline $25.0 \%$ & $93.1 \%$ & $44.4 \%$ & $64.3 \%$ & $85.7 \%$ \\
\hline $20.0 \%$ & $93.1 \%$ & $57.1 \%$ & $64.3 \%$ & $90.9 \%$ \\
\hline $15.0 \%$ & $93.1 \%$ & $68.8 \%$ & $64.3 \%$ & $94.3 \%$ \\
\hline $10.0 \%$ & $93.1 \%$ & $79.7 \%$ & $64.3 \%$ & $96.7 \%$ \\
\hline
\end{tabular}

Table 3. Variation of accuracy for VNT based on different simulated prevalence.

\begin{tabular}{|c|c|c|c|c|}
\hline & \multicolumn{2}{|c|}{ Any Esophageal Varices } & \multicolumn{2}{|c|}{ Varices Needing Treatment (VNT) } \\
\hline & OR $[95 \% \mathrm{CI}]$ & $\mathbf{p}$ & OR $[95 \% \mathrm{CI}]$ & $\mathbf{p}$ \\
\hline Aspartate Aminotransferase & $0.998[0.973-1.004]$ & 0.134 & $0.997[0.971-1.025]$ & 0.851 \\
\hline Alanine Aminotransferase & $1.015[0.992-1.040]$ & 0.209 & $1.008[0.974-1.043]$ & 0.651 \\
\hline Platelet Count & $1.000[1.000-1.000]$ & 0.133 & $1.000[1.000-1.000]$ & 0.574 \\
\hline Spleen Diameter & $1.033[1.008-1.057]$ & 0.009 & $1.021[0.979-1.065]$ & 0.337 \\
\hline Portal Vein Diameter & $1.053[0.813-1.363]$ & 0.697 & $0.972[0.548-1.722]$ & 0.922 \\
\hline Hemoglobin & $0.884[0.633-1.234]$ & 0.468 & $0.789[0.511-1.218]$ & 0.284 \\
\hline Spleen Elastography & $3.976[1.531-10.320]$ & 0.005 & $1360.730[34.183-54166.263]$ & 0.000 \\
\hline Liver Elastography & $1.468[0.621-3.473]$ & 0.382 & $0.955[0.258-3.538]$ & 0.945 \\
\hline \multirow[t]{2}{*}{ Constant } & 0.01 & 0.038 & 0 & 0.003 \\
\hline & \multicolumn{2}{|c|}{$\begin{array}{l}\text { AUROC }=0.845[0.778-0.911] \\
\text { Goodness of fit }=38.009 \\
p<0.001\end{array}$} & \multicolumn{2}{|c|}{$\begin{array}{l}\text { AUROC }=0.974[0.950-0.997] \\
\text { Goodness of fit }=75.708, p<0.001\end{array}$} \\
\hline
\end{tabular}

Table 4. The multivariate analysis results.

of portal pressure is a constant concern that influences the outcomes in both stages of cirrhosis: compensated or decompensated. HVPG and upper endoscopy are the gold-standard techniques for the diagnosis of portal hypertension and esophageal varices. However, their invasiveness makes them rarely accepted by patients. The non-invasive tests are able to provide similar information and are more welcomed. Previous studies, that have used HVPG as a reference, have shown a significant association between the LSM values and the risk of portal hypertension development in cirrhotic patients, by assessing the liver stiffness with the help of transient elastography ${ }^{26-32}$. Also, there are several studies that analyzed the predictive value of TE for esophageal varices and concluded that there is a significant correlation between TE values and the existence of esophageal varices ${ }^{33-37}$. However, in severe cirrhosis, the link between LSM and HVPG lessens as a result of cumulating factors, such as mechanical changes in the liver, mainly fibrosis, and the dynamic feature of $\mathrm{PH}$ (intrahepatic vasoconstriction and 


\begin{tabular}{|l|l|l|l|}
\hline & $\begin{array}{l}\text { Any Esophageal } \\
\text { Varices }\end{array}$ & $\begin{array}{l}\text { Varices Needing } \\
\text { Treatment (VNT) }\end{array}$ & Overall \\
\hline Alcoholic & $3.47 \mathrm{~m} / \mathrm{s}$ & $4.04 \mathrm{~m} / \mathrm{s}$ & $3.31 \mathrm{~m} / \mathrm{s}$ \\
\hline Hepatitis B & $3.05 \mathrm{~m} / \mathrm{s}$ & $3.67 \mathrm{~m} / \mathrm{s}$ & $2.97 \mathrm{~m} / \mathrm{s}$ \\
\hline Hepatitis C & $3.40 \mathrm{~m} / \mathrm{s}$ & $3.94 \mathrm{~m} / \mathrm{s}$ & $3.14 \mathrm{~m} / \mathrm{s}$ \\
\hline Other & $3.63 \mathrm{~m} / \mathrm{s}$ & $4.15 \mathrm{~m} / \mathrm{s}$ & $3.05 \mathrm{~m} / \mathrm{s}$ \\
\hline Test & $\begin{array}{l}\text { Independent Samples } \\
\text { Kruskal-Wallis }\end{array}$ & $\begin{array}{l}\text { Independent Samples } \\
\text { Kruskal-Wallis }\end{array}$ & $\begin{array}{l}\text { Independent Samples } \\
\text { Kruskal-Wallis }\end{array}$ \\
\hline p-value & 0.078 & 0.383 & 0.301 \\
\hline
\end{tabular}

Table 5. Assessment of differences between different etiologies of cirrhosis.

splanchnic vasodilation) that cannot be evaluated by $\mathrm{TE}^{38}$. A recent meta-analysis, that evaluated the performance of TE for detecting esophageal varices in cirrhotic patients, concluded that TE is not suitable for implementation in the clinical practice due to varying cut-off values and different etiologies ${ }^{39}$. The morphological changes of the spleen in portal hypertension, such as the vascular congestion angiogenesis, the lymphoid hyperplasia and the fibrosis have justified the use of spleen stiffness as another surrogate parameter of $\mathrm{PH}$ in cirrhosis ${ }^{40,41}$.

As a result, our study wanted to determine the predictive value of SSM measurement through virtual touch quantification, in the assessment of the existence and gravity of esophageal varices (EV) in patients suffering from cirrhosis of different etiologies. Upper digestive endoscopy was used as a comparison for the efficiency of this method. Spleen stiffness measurements were successfully carried out in all enrolled patients, resulting in a wide range of values that varied between $2.18 \mathrm{~m} / \mathrm{s}$ and $4.77 \mathrm{~m} / \mathrm{s}$. The results showed a stepwise increase in splenic stiffness with the increasing size of the varices $(p<0.001)$. The accuracy of SSM in predicting the presence of EV was demonstrated to be fair - good, with an AUROC of 0.776 and a good sensitivity and specificity (Se 72\%, Sp 71\%). The values for confirming or excluding the presence of the varices were: $\geq 3.50 \mathrm{~m} / \mathrm{s}$ and $<2.50 \mathrm{~m} / \mathrm{s}$ respectively. There are many studies that deemed the importance of spleen stiffness as being equivalent to that of the endoscopy in predicting esophageal varices ${ }^{5,17,42}$. The majority of these used TE when performing SSM (TE-SSM) ${ }^{43-47}$.

There are at least two limitations for TE-SSM: the high percentage of failed measurements and the variability of the values with respect to the etiology of the liver diseases. In addition, there is one more limitation regarding the upper limit of the cut-off value of $75 \mathrm{kPa}$, considering that SSM frequently exceeds this value $5,42,45,46$.

There are several studies that performed ARFI for the assessment of spleen stiffness, alone $\mathrm{e}^{10,18,48-50}$ or in comparison with $\mathrm{TE}^{5}$. Only two of those took HVPG as a reference for evaluating the spleen stiffness measurement performance ${ }^{51,52}$. Both of them revealed the remarkable accuracy of SSM in predicting clinically relevant portal pressure values. Most of the studies were aimed at evaluating the connection between SSM, measured through ARFI, and the presence and stage of the esophageal varices. As demonstrated in our study, there is a considerable correlation between ARFI-SSM and the existence of EVs in patients suffering from cirrhosis. Also, our study showed no significant differences between the optimal cut-off values of ARFI-SSM in predicting the existence of varices: $3.10 \mathrm{~m} / \mathrm{s}^{48} 3.18 \mathrm{~m} / \mathrm{s}^{51} / 3.16 \mathrm{~m} / \mathrm{s}^{5}$ and $3.02 \mathrm{~m} / \mathrm{s}$ in our study. With regard to the performance of ARFI-SSM in predicting the severity of $\mathrm{PH}$, expressed as the size of varices and the risk of bleeding, our results showed an excellent performance, AUROC $=0.972$, in recognizing patients with varices needing treatment. The cut-off values of ARFI-SSM to rule out and rule in the varices at risk for bleeding were $<3.20$ and $\geq 3.80 \mathrm{~ms} / \mathrm{s}$ respectively. For a cut-off value of $3.20 \mathrm{~m} / \mathrm{s}$ the predictive negative value is remarkable (99\%) for excluding the cases with varices needing treatment. Another advantage of ARFI, underlined by all these studies, is the high feasibility of this method, without measurement failures like in the case of TE-SSM. However, there is one study that did not find ARFI-SSM to be a good parameter in evaluating the existence and gravity of $\mathrm{EV}^{53}$. On the other hand, the same group developed a predictive score that analyzed LSM and SSM, using ARFI measurements, in cirrhotic patients associating ascites, with a good accuracy in anticipating significant $\mathrm{EV}^{50}$.

One concern was raised regarding SSM as a complementary method in evaluating the existence of EVs in patients suffering from cirrhosis: the loss of correlation in alcohol induced cirrhosis ${ }^{49}$. Without a clear reason for this result, the authors have considered the smaller dimension of the spleen that characterized alcoholic cirrhosis to be a probable cause, as compared to viral or non-alcoholic steatohepatitis-cirrhosis. Our study did not confirm this hypothesis, because it found no differences between the diverse etiology of cirrhosis, including the one induced by alcohol $(\mathrm{p}=0.301)$.

Another purpose of our study was to analyze the other parameters that were linked to the existence and severity of EV, in patients suffering from cirrhosis. In this regard, bivariate analysis showed a significant correlation, alongside SSM, of the platelet number, splenic size, portal vein diameter, AST values and ARFI - liver elasticity $(\mathrm{p}<0.001)$. All these parameters were related not only to the presence and size of the varices, but also to the varices needing treatment. However, after multivariate analysis, SSM remained the only parameter that was highly associated with the presence and size of EVs and also with their risk of bleeding. There was another independent variable associated only with one, but not with all characteristics of esophageal varices: the splenic diameter used for detecting the presence of EV $(\mathrm{p}=0.009)$. However, SSM was indisputably the strongest predictor for all endoscopic aspects of $\mathrm{EV}$, from their presence, to the detection of varices needing treatment. Other studies also found ARFI-SSM to be a unique independent parameter for EV prediction, according to multivariate analysis ${ }^{48,49}$.

One limitation of our study is the uneven distribution of cirrhosis etiology, having only a few cases with NASH-cirrhosis, autoimmune and biliary cirrhosis. Therefore, it is difficult to assess the efficacy of splenic stiffness in different etiologies of cirrhosis. Another limitation is the lack of a standard protocol for performing the measurements for spleen stiffness. Usually, the measurements were made $2 \mathrm{~cm}$ under the splenic capsule, 
mostly in the middle part of the spleen. In the study of Rizo et al. ${ }^{41}$ the measurements were extensively performed throughout the entire splenic parenchyma, in order to be more representative.

However, we consider that ARFI spleen stiffness measurement is an optimal method to use in the clinical practice, for the screening of cirrhotic patients, for esophageal varices. By easily and reliably diagnosing high-risk varices, ARFI can discriminate variceal bleeders from non-bleeders. We believe that our study may contribute to recognizing splenic stiffness as a novel parameter and an unlimited tool for gastroenterologists to use in the screening of the cirrhotic population, reducing the number of endoscopic examinations and, as a result, improving the Baveno VI criteria.

Received: 1 May 2019; Accepted: 9 October 2019;

Published online: 07 November 2019

\section{References}

1. Garcia-Tsao, G. \& Bosch, J. Management of varices and variceal hemorrhage in cirrhosis. N Engl J Med. 362, 823-832 (2010).

2. Garcia-Tsao, G., Sanyal, A. J., Grace, N. D. \& Carey, W. Practice Guidelines Committee of the American Association for the Study of Liver D, Practice Parameters Committee of the American College of G. Prevention and management of gastroesophageal varices and variceal hemorrhage in cirrhosis. Hepatology 46, 922-938 (2007).

3. Bosch, J., Abraldes, J. G., Berzigotti, A. \& Garcia-Pagan, J. C. The clinical use of HVPG measurements in chronic liver disease. Nat Rev Gastroenterol Hepatol. 6, 573-582 (2009).

4. de Franchis, R. \& Baveno, V. I. F. Expanding consensus in portal hypertension: Report of the Baveno VI Consensus Workshop: Stratifying risk and individualizing care for portal hypertension. J Hepatol. 63, 743-752 (2015).

5. Kim, H. Y. et al. The Role of Spleen Stiffness in Determining the Severity and Bleeding Risk of Esophageal Varices in Cirrhotic Patients. Medicine (Baltimore) 94, e1031 (2015).

6. Takuma, Y. et al. Measurement of Spleen Stiffness With Acoustic Radiation Force Impulse Imaging Predicts Mortality and Hepatic Decompensation in Patients With Liver Cirrhosis. Clin Gastroenterol Hepatol. 15, 1782-1790 (2017).

7. Buechter, M. et al. Spleen and Liver Stiffness Is Positively Correlated with the Risk of Esophageal Variceal Bleeding. Digestion 94, 138-144 (2016)

8. Singh, S. et al. Accuracy of spleen stiffness measurement in detection of esophageal varices in patients with chronic liver disease: systematic review and meta-analysis. Clin Gastroenterol Hepatol. 12, 935-945 e934 (2014).

9. Llop, E. et al. Validation of noninvasive methods to predict the presence of gastroesophageal varices in a cohort of patients with compensated advanced chronic liver disease. J Gastroenterol Hepatol. 32, 1867-1872 (2017).

10. Takuma, Y. et al. Portal Hypertension in Patients with Liver Cirrhosis: Diagnostic Accuracy of Spleen Stiffness. Radiology 279, 609-619 (2016).

11. Roccarina, D., Rosselli, M., Genesca, J. \& Tsochatzis, E. A. Elastography methods for the non-invasive assessment of portal hypertension. Expert Rev Gastroenterol Hepatol. 12, 155-164 (2018).

12. Colecchia, A., Ravaioli, F., Marasco, G., Festi, D. Spleen Stiffness by Ultrasound Elastography. In: Berzigotti, A., Bosch, J. Diagnostic Methods for Cirrhosis and Portal Hypertension, 113-137 (2018)

13. Karagiannakis, D.S., et al Role of Spleen Stiffness Measurement by 2D-Shear Wave Elastography in Ruling Out the Presence of HighRisk Varices in Cirrhotic Patients. Dig Dis Sci, (2019)

14. Colecchia, A. et al. A combined model based on spleen stiffness measurement and Baveno VI criteria to rule out high-risk varices in advanced chronic liver disease. J Hepatol. 69, 308-317 (2018).

15. Dietrich, C. F. et al. EFSUMB Guidelines and Recommendations on the Clinical Use of Liver Ultrasound Elastography, Update 2017 (Long Version). Ultraschall in Med. 38, e16-e47 (2017).

16. Colecchia, A. et al. Spleen stiffness measurement can predict clinical complications in compensated HCV-related cirrhosis: a prospective study. J Hepatol. 60, 1158-1164 (2014).

17. Wong, G. L. et al. Measuring spleen stiffness to predict varices in chronic hepatitis B cirrhotic patients with or without receiving non-selective beta-blockers. J Dig Dis. 17, 538-546 (2016).

18. Ye, X. P. et al. Liver and spleen stiffness measured by acoustic radiation force impulse elastography for noninvasive assessment of liver fibrosis and esophageal varices in patients with chronic hepatitis B. J Ultrasound Med. 31, 1245-1253 (2012).

19. Hirooka, M. et al. Splenic elasticity measured with real-time tissue elastography is a marker of portal hypertension. Radiology 261, 960-968 (2011)

20. Chen, Y. P. et al. Is transient elastography valuable for high-risk esophageal varices prediction in patients with hepatitis-B-related cirrhosis? J Gastroenterol Hepatol. 27, 533-539 (2012).

21. Elkrief, L. et al. Prospective comparison of spleen and liver stiffness by using shear-wave and transient elastography for detection of portal hypertension in cirrhosis. Radiology 275, 589-598 (2015).

22. Cassinotto, C. et al. Liver and spleen elastography using supersonic shear imaging for the non-invasive diagnosis of cirrhosis severity and oesophageal varices. Dig Liver Dis. 47, 695-701 (2015).

23. Heidelbaugh, J. J. \& Bruderly, M. Cirrhosis and chronic liver failure: part I. Diagnosis and evaluation. Am Fam Physician 74, 756-762 (2006).

24. Romania MoHo. Annex 12 - Cirrhosis and complications (Anexa 12 - Ciroza hepatica si complicatiile cirozei hepatice).

25. Augustin, S. et al. Expanding the Baveno VI criteria for the screening of varices in patients with compensated advanced chronic liver disease. Hepatology 66, 1980-1988 (2017).

26. Bureau, C. et al. Transient elastography accurately predicts presence of significant portal hypertension in patients with chronic liver disease. Aliment Pharmacol Ther. 27, 1261-1268 (2008).

27. Carrion, J. A. et al. Transient elastography for diagnosis of advanced fibrosis and portal hypertension in patients with hepatitis C recurrence after liver transplantation. Liver Transpl. 12, 1791-1798 (2006).

28. Reiberger, T. et al. Vienna Hepatic Hemodynamic Lab. Non-selective betablocker therapy decreases intestinal permeability and serum levels of LBP and IL-6 in patients with cirrhosis. J Hepatol. 58, 911-921 (2013).

29. Lemoine, M. et al. Liver stiffness measurement as a predictive tool of clinically significant portal hypertension in patients with compensated hepatitis C virus or alcohol-related cirrhosis. Aliment Pharmacol Ther. 28, 1102-1110 (2008).

30. Schwabl, P. et al. New reliability criteria for transient elastography increase the number of accurate measurements for screening of cirrhosis and portal hypertension. Liver Int. 35, 381-390 (2015).

31. Sanchez-Conde, M. et al. Use of transient elastography (FibroScan(R)) for the noninvasive assessment of portal hypertension in HIV/HCV-coinfected patients. J Viral Hepat. 18, 685-691 (2011).

32. Berzigotti, A. et al. Elastography, spleen size, and platelet count identify portal hypertension in patients with compensated cirrhosis. Gastroenterology 144, 102-111 (2013).

33. Castera, L. et al. Early detection in routine clinical practice of cirrhosis and oesophageal varices in chronic hepatitis C: comparison of transient elastography (FibroScan) with standard laboratory tests and non-invasive scores. J Hepatol. 50, 59-68 (2009). 
34. Kazemi, F. et al. Liver stiffness measurement selects patients with cirrhosis at risk of bearing large oesophageal varices. J Hepatol. 45 , 230-235 (2006).

35. Sporea, I., Ratiu, I., Bota, S., Sirli, R. \& Jurchis, A. Are different cut-off values of liver stiffness assessed by transient elastography according to the etiology of liver cirrhosis for predicting significant esophageal varices? Med Ultrason. 15, 111-115 (2013).

36. Saad, Y., Said, M., Idris, M. O., Rabee, A. \& Zakaria, S. Liver stiffness measurement by fibroscan predicts the presence and size of esophageal varices in egyptian patients with HCV related liver cirrhosis. JClin Diagn Res. 7, 2253-2257 (2013).

37. Nguyen-Khac, E. et al. Noninvasive diagnosis of large esophageal varices by Fibroscan: strong influence of the cirrhosis etiology. Alcohol Clin Exp Res. 34, 1146-1153 (2010).

38. Vizzutti, F., Arena, U., Rega, L. \& Pinzani, M. Non invasive diagnosis of portal hypertension in cirrhotic patients. Gastroenterol Clin Biol. 32, 80-87 (2008).

39. Pu, K. et al. Diagnostic accuracy of transient elastography (FibroScan) in detection of esophageal varices in patients with cirrhosis: A meta-analysis. World J Gastroenterol. 23, 345-356 (2017).

40. Bolognesi, M., Merkel, C., Sacerdoti, D., Nava, V. \& Gatta, A. Role of spleen enlargement in cirrhosis with portal hypertension. Dig Liver Dis. 34, 144-150 (2002).

41. Mejias, M. et al. Relevance of the mTOR signaling pathway in the pathophysiology of splenomegaly in rats with chronic portal hypertension. J Hepatol. 52, 529-539 (2010).

42. Calvaruso, V. et al. Modified spleen stiffness measurement by transient elastography is associated with presence of large oesophageal varices in patients with compensated hepatitis C virus cirrhosis. J Viral Hepat. 20, 867-874 (2013).

43. Colecchia, A. et al. Measurement of spleen stiffness to evaluate portal hypertension and the presence of esophageal varices in patients with HCV-related cirrhosis. Gastroenterology 143, 646-654 (2012).

44. Sharma, P. et al. Spleen stiffness in patients with cirrhosis in predicting esophageal varices. Am J Gastroenterol. 108, 1101-1107 (2013).

45. Fraquelli, M. et al. Feasibility and reproducibility of spleen transient elastography and its role in combination with liver transient elastography for predicting the severity of chronic viral hepatitis. J Viral Hepat. 21, 90-98 (2014).

46. Stefanescu, H. et al. Spleen stiffness measurement using Fibroscan for the noninvasive assessment of esophageal varices in liver cirrhosis patients. J Gastroenterol Hepatol. 26, 164-170 (2011).

47. Stefanescu, H. et al. Non-invasive menage a trois for the prediction of high-risk varices: stepwise algorithm using lok score, liver and spleen stiffness. Liver Int. 35, 317-325 (2015).

48. Rizzo, L. et al. A new sampling method for spleen stiffness measurement based on quantitative acoustic radiation force impulse elastography for noninvasive assessment of esophageal varices in newly diagnosed HCV-related cirrhosis. Biomed Res Int. 2014, 365982 (2014).

49. Park, J. et al. Is the spleen stiffness value acquired using acoustic radiation force impulse (ARFI) technology predictive of the presence of esophageal varices in patients with cirrhosis of various etiologies. Med Ultrason. 18, 11-17 (2016).

50. Bota, S. et al. Can ARFI elastography predict the presence of significant esophageal varices in newly diagnosed cirrhotic patients? Annals of hepatology 11, 519-525 (2012).

51. Takuma, Y. et al. Measurement of spleen stiffness by acoustic radiation force impulse imaging identifies cirrhotic patients with esophageal varices. Gastroenterology 144, 92-101 (2013).

52. Attia, D. et al. Evaluation of Liver and Spleen Stiffness with Acoustic Radiation Force Impulse Quantification Elastography for Diagnosing Clinically Significant Portal Hypertension. Ultraschall Med. 36, 603-610 (2015).

53. Bota, S. et al. Spleen assessment by Acoustic Radiation Force Impulse Elastography (ARFI) for prediction of liver cirrhosis and portal hypertension. Med Ultrason. 12, 213-217 (2010).

\section{Author contributions}

Fierbinteanu-Braticevi C. designed the study; Tribus L., Peagu R., Petrisor A., Oprea G., Purcareanu A. contributed to acquisition of data; Baicus C., Moldoveanu A., Cretoiu D. analyzed the data; FierbinteanuBraticevi C., Moldoveanu A., Cretoiu D. drafted the manuscript; Baicus C., Moldoveanu A., Purcareanu A. performed the statistical analysis, Fierbinteanu-Braticevi C., Moldoveanu A., Peagu R., Pasternak A. revised the manuscript's intellectual content and Fierbinteanu-Braticevi C. provided administrative, technical support and study supervision.

\section{Competing interests}

The authors declare no competing interests.

\section{Additional information}

Correspondence and requests for materials should be addressed to D.C.

Reprints and permissions information is available at www.nature.com/reprints.

Publisher's note Springer Nature remains neutral with regard to jurisdictional claims in published maps and institutional affiliations.

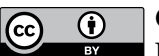

Open Access This article is licensed under a Creative Commons Attribution 4.0 International

License, which permits use, sharing, adaptation, distribution and reproduction in any medium or format, as long as you give appropriate credit to the original author(s) and the source, provide a link to the Creative Commons license, and indicate if changes were made. The images or other third party material in this article are included in the article's Creative Commons license, unless indicated otherwise in a credit line to the material. If material is not included in the article's Creative Commons license and your intended use is not permitted by statutory regulation or exceeds the permitted use, you will need to obtain permission directly from the copyright holder. To view a copy of this license, visit http://creativecommons.org/licenses/by/4.0/.

(C) The Author(s) 2019 